\title{
Kasenova M.B.
}

\section{Internationalization possibilities and perspectives in trans-border Internet governance: the legal context}

\begin{abstract}
This article analyzes the Announcement of the U.S. Commerce Department's National Telecommunications and Information Administration (from March 14, 2014) about the intentions of the USA Government to pass the governance of the implementation of the functions of the IANA to the "global multistakeholders; the outcome documents of the NET-Mundial Global Multistakeholder Meeting on the Future of Internet Governance (April 2014, São Paulo, Brazil), and also the Panel on Global Internet Cooperation and Governance Mechanisms (May 2014). In the author's opinion, the implementation of suggestions and measures, specified by the enumerated documents, can radically change the governance of the technological infrastructure of the Internet, and have a substantial influence on the internationalization of the trans-border Internet governance. Now it is fundamentally important to discuss the ecosystem of Internet governance, the high-level structure of which is outlined in the summary documents produced by the NET-Mundial 2014, where the principles of Internet governance and the roadmap of the future evolution of the ecosystem of Internet governance was formulated.
\end{abstract}

Keywords: Internet, International Assigned Numbers Authority, the functions of the IANA, Internet governance, multistakeholder participation, stakeholders, NETMundial, Panel, global Internet cooperation, Internet governance mechanisms.

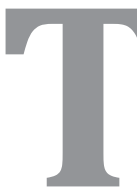

hree key events of spring 2014 can apparently have a substantial influence on the set of problems that this article covers. Firstly, it's the announcement of the U.S. Commerce Department's National Telecommunications and Information Administration from March $2014^{1}$, that is critically important, and its

\footnotetext{
${ }^{1}$ URL: http://www.ntia.doc.gov/press-release/2014/ntia-
}

implementation can change the whole existing configuration of trans-border Internet control. Secondly, the NET-Mundial Global Multi-stakeholder Meeting on the Future

announces-intent-transition-key-internet-domain-namefunctions; and also _ URL: press@ntia.doc.gov (access date 09.08.2014); - URL: http://www.inta.org/INTABulletin/Pages/ USDepartmentofCommerceAnnouncesIntenttoTransitionKeyI nternetDomainNameFunctions.aspx; -URL: https://www.afnic. $\mathrm{fr} / \mathrm{fr} /$ ressources/blog/l-elephant-iana-est-dans-la-salle-2.html. 
of Internet Governance, which was held in April 2014 in São Paulo (Brazil) ${ }^{1}$, and its results. Thirdly, the end of the work of the Panel on Global Internet Cooperation and Governance Mechanisms ${ }^{2}$ and the resulting documents, approved by this panel in May, 2014.

\section{I.}

The Announcement of the U.S. Commerce Department's National Telecommunications and Information Administration (NTIA), made on March 14, 2014, further - the "NTIA Announcement, March 14, 2014", expresses the desire of the USA Government to change its role in the governance of the technological infrastructure of the Internet radically, and to pass the control of the implementation of the IANA function to the Global Multistakeholder Community. This process is to be finished in September 2015. An understanding of the NTIA Announcement of 14.03.2014 is connected to the following:

The trans-border functioning of the Internet is secured by the Domain Name System (DNS) control; the coordination of the global IP-address and port numbers pool distribution to regional Internet registers; the provision of storage and the distribution of names and numbers of register Internetprotocols ${ }^{3}$. The above-mentioned functions have a key meaning for the Internet, and as the Internet Assigned Numbers Authority (IANA) provides the technical coordination of their implementation on global level, they are named as the functions of the IANA. The implementation of the functions of the IANA and their content are the principal questions of trans-border governance of the technological infrastructure of the Internet, and the meaning of the Functions of the IANA for the

\footnotetext{
${ }^{1}$ URL: http://www.netmundial.br

${ }^{2}$ URL: http://internetgovernancepanel.org/

${ }^{3}$ URL: http://www.internetsociety.org/internet/what-internet/ history-internet/brief-history-internet-related-networks
}

Internet is so big that they can be called The Holy Grail for the government in the context of decision- making in Internet governance 4 .

The coordination of the functioning of unique Internet identifiers and the control of the Domain Name System were initially performed under the control of the USA Government. In 1977, the USA Government, represented by the United States Department of Defense, signed a contract about the implementation of the functions of the IANA with the University of Southern California and the Information Science Institute (USAC/ISI). ${ }^{5}$ In fact, the above-mentioned contract became the basis of the contractual, legal format of the implementation of the functions of the IANA, which continues till the present time $^{6}$. The signing of the contract with the University of Southern California Information Science Institute was related to the fact that the Institute had a team of technical experts (the IANA Team) headed by one of the founding fathers of the Internet, Jon Postel ${ }^{7}$.

\footnotetext{
${ }^{4}$ URL: http://linguasynaptica.com/how-do-you-solve-aproblem-like-iana/ (load date 09.09.2014).

${ }^{5}$ URL: https://www.princeton.edu/ achaney/tmve/wiki100k/ docs/Internet_Assigned_Numbers_Authority.html

${ }^{6}$ See also "Statement of Policy on the Management of Internet Names and Address" for IANA functions implementation.URL: http://www.ntia.doc.gov/federal-register-notice/1998/ statement-policy-management-internet-names-and-addresses ${ }^{7}$ In the nineties there was sort of a "war for power" going on between the "founding fathers of the Internet, acclaimed by the technological community, such as Jon Postel, and the USA Government, which was entirely controlling the domain names and the distribution of IP addresses. There is quite a remarkable episode during this period: Jon Postel was apparently trying to take control of the domain names and addresses distribution system, and asked the root server operators to recognize the server that belonged to him as the key tool in the domain name and address distribution system. All the operators, probably because of respect to Jon Postel personally, instantly granted his request. Ira C. Magaziner, the USA Government official, announced that she was going to sue Jon Postel and his employer, if he did not "give back" the domain names distribution system into the control of the USA Government. See MayerSchönbergerV., ZiewitzM.JeffersonRebuffed: The United States and the Future of Internet Governance // Columbia Science and Technology Law Review. 188. (2007)._ URL: http://www. stlr.org/html/volume8/schoenbergerintro.php; and also-URL: http://www.livinginternet.com/i/iw_mgmt_iana.htm
} 
In 1997 the USA Government made a decision to change its administrative role in the DNS distribution, and in 1998 the Internet Corporation for Assigned Names and Numbers (ICANN) was created, as "the ICANN Corporation", or "ICANN". The ICANN Corporation is a legal entity, created within the type of Non-Profit Organization in accordance with the state laws of California, USA. In fact, the creation of the ICANN Corporation formally concluded the transition of the DNS administration from the USA Government, represented by the Commerce Department, to a private sector organization ${ }^{1}$.

After the ICANN Corporation was created, the IANA Administration was included into its internal organizational structure, and the USA Government made a contract with the ICANN Corporation about the implementation of the IANA functions (which became the "ICANN/USA Government Contract"). It is notable that the USA Government, specifically the U. S. Commerce Department's National Telecommunications and Information Administration represented one of the sides of the ICANN/USA Government Contract. The first ICANN/ USA Government Contract was signed in 2000. Later this contract was renewed, and regularly changed. The contract time of the current ICANN/USA Government Contract was running to its end on September 30, 2012, but on July 3, 2012 the U. S. Commerce Department's National Telecommunications and Information Administration announced that the implementation time of the ICANN/ USA Government Contract should be prolonged for seven more years, with the possibility of two stages of options. The first op-

\footnotetext{
${ }^{1}$ The information document published by the USA Government declared that the USA Government would overlap the DNS administration, which will allow the private sector to take the lead. See details: URL: http://www.ntia.doc.gov/ntiahome/domainname/6_5_98dns. htm\#N_16_
}

tion of the ICANN/USA Government Contract is set from October 1, 2012 to September 30, 2015; the second option, from October 1 , 2017 to September 30, 2019. ${ }^{2}$

The ICANN/USA Government Contract reaffirms the functions of the IANA, describes their content and regulates their implementation ${ }^{34}$. Within this article, we see it reasonable to refer the key arguments of this document

If we start from the regulatory legal content of the ICANN/USA Government Contract, its more accurate translation to Russian will be - "governmental contract on the contractor work execution of the implementation of the functions of the IANA". At the same time by the terms of the contract, the ICANN Corporation acts together with the IANA Administration and is a Contractor. The Contractor ICANN/IANA implements the functions of the IANA, reports on the completion of these functions, and provides this information to all the Stakeholders in the Internet-community. The assignment of the last term is connected to the fact that the ICANN/USA Government Contract assumes the need to solve the problems of the Internet, taking into account multistakeholder mechanisms, and the following internationalization process of the IANA functions implementation.

The most important term for the implementation of the functions of the IANA is that the Contractor ICANN/IANA is limited in its rights: it can't involve subcontractors. The ICANN/USA Government

\footnotetext{
${ }^{2}$ URL: http://www.ntia.doc.gov/files/ntia/publications/ sf_26_pg_1-2-final_award_and_sacs.pdf; and also URL:http:// www.vharris@ntia.doc.gov; URL: http://www.ntia.doc.gov/ press-release/2012/commerce-department-awards-contractmanagement-key-internet-functions-icann

${ }^{3}$ For the IANA functions content see, for example, Kasenova M. B. Internet governance International legal mechanism - SPb.: Center of humanities initiatives, 2012 P.55-65.

${ }^{4}$ See URL: http://www.iana.org/assignments/iana-ipv6-specialregistry/iana-ipv6-special-registry.xhtml
} 
contract defines the "control" criterion. The contractual legal definition of the "control" criterion helps to define the true state affiliation of an entity, gives the state the ability to defend the interests of its entities, among other things by regulating the question of the access of foreign institutions to its territory, or by setting a specific legal regime towards the activities of foreign entities etc. ${ }^{1}$ The definition of "control" criterion also correlates with the statement of the ICANN/USA Government contract about the USA Government retaining the right to control the premises, systems and processes of all power and operating components, used to fulfill all the contractual obligations and requirements, related to the implementation of the functions of the IANA.

The ICANN/USA Government Contract foresees that the works related to any aspects of the implementation of the functions of the IANA can be carried out only by organizations registered in the USA, or fully under control of the USA companies, or organized and created by the laws of the USA states, and the District of Columbia, USA. It is assumed that the contractual legal definition of the "control" criterion by the ICANN/USA Government Contract creates defined legal regulation of the IANA functions implementation: firstly, by defining the nature of parties of legal relations, secondly, by resolving the question of applicable law, thirdly, by creating legal grounds for American court jurisdiction. It should be noted that the questions of the jurisdiction for the regulation of relations on the Internet are most complicated, and the legislation in the field of jurisdiction, including the problems of laws and juris-

\footnotetext{
${ }^{1}$ About the "control" criterion in the doctrine of international private law, see, for example: Asoskov A. V. Legal forms of legal entities' participation in international commerce. M.: Statut. 2003; Voznesenskaya N. N. Legal Entities in international private law of Russia and EU // Izvestiya vuzov Law studies. 2009. № 3 and others.
}

dictions clashes, was developed in detail specifically in the USA ${ }^{2}$.

The contents of the ICANN/USA Government Contract, the nature of its parties and the defined order of their interactions give the grounds for qualifying it as a public agreement, containing elements of private law regulation. Public agreements, unlike private law agreements, have a specific goal and functional direction, which appears in particular in the fact that by signing such agreements the state is realizing public interests and is not pursuing a profit goal ${ }^{3}$. The non-profit nature of the ICANN/USA Government contract is apparent from its terms: the IANA functions are implemented "... at no cost, $\$ 0.00$ time and a material contract"4.

Besides the detailed regulations of the implementation of the technical IANA functions, the ICANN/USA Government Contract recognizes the need to develop tight constructive working relations with all the stakeholders, to ensure the quality implementation of the IANA functions. The stakeholders of the ICANN/

\footnotetext{
${ }^{2}$ Savelyev A.I. Electronic commerce in Russia and abroad: legal regulation.- M.: Statut, 2014 P. 48. See also: MentheD. Jurisdiction In Cyberspace: A Theory of International Spaces // Michigan Telecommunication Technical Revue. № 4 (69). URL: http://www.law.umich.edu/mttlr/vol-four/menthe.html; Post D. Personal Jurisdiction on the Internet: An Outline for the Perplexed // Temple University Law School. 1998.

${ }^{3}$ In USA, Public Contracts are regulated by section 41 of the USCode, U.S.C.). - URL: http://law.onecle.com/uscode/41/ index.html. See details, for example: Barry L. McVay. The Essential Guide to Federal Contracts. - URL: http://www. loot.co.za/product/barry-l-mcvay-the-essential-guide-tofederal-contracts/mchz-371-g410; Government Contract Law, by the Section of Public Contract Law of the American Bar Association (2007). - URL: http://www.americanbar.org/ content/dam/aba/publications/public_contract_law_journal/ pc_writing_competition/2014_writing_competition_rules. authcheckdam.pdf; Edward J. Vernon. Recommended Books for Government Contracting Professionals. - URL: http:// www.wifcon.com/anal/RecommendedReading.pdf; - URL: http://blogs.loc.gov/law/2013/06/government-contracts-abeginners-guide/

${ }^{4}$ Section "C" of the ICANN/USA Government Contract URL: http://www.ntia.doc.gov/files/ntia/publications/sf_26_ pg_1-2-final_award_and_sacs.pdf
} 
USA Government Contract include the Internet Engineering Task Force (IETF) and the Internet Architecture Board (IAB) of the Internet Society (ISOC), the Regional Internet Registries $(R I R)$, the Top-level domain $(T L D)$ operators, governments, civil society, that provide various pieces of information and data connected to the development of model politics for the Domain Name System $(D N S)^{1}$.

The specifics of the IANA functions are that they should be implemented in cooperation with the protocols and standards of network architecture and the technology of the Internet, and at the same time the main functions are that of parameter definition for the protocol used in Internet standards, developed by the Internet Engineering Task Force (IETF) of the Internet Society (ISOC), further - the IETF group. As the IETF group mission is connected to "the optimization of Internet functioning through the creation of high-quality technical documents and protocols, which influence the design, use and governance of the Internet" ${ }^{\prime 2}$, it cooperates with all organizations and structures, supplying the functioning of a technological infrastructure for the Internet, and, above all, with the ICANN Corporation, which implements the functions of the IANA.

The legal result of the above-mentioned specifics is that the implementation of the IANA functions assumes "technical provision", which is regulated by the agreement signed between the IETF Group and the ICANN Corporation - the IETF/ICANN Memorandum of Understanding Concerning the Technical Work of the Internet Assigned Number Authority, as in the IETF/ICANN Memorandum. The current IETF/ICANN Memorandum, signed on March 1, 200033,

\footnotetext{
${ }^{1}$ URL: http://linguasynaptica.com/how-do-you-solve-aproblem-like-iana/

2"A Mission Statement for the IETF”. - URL: http://www.ietf. org/rfc/rfc3935.txt

${ }^{3}$ http://www.icann.org/en/general/ietf-icann-mou-01mar00.htm
}

is changed and expanded each year, starting from 2007 with the acceptance of the corresponding Supplemental Agreement. Now the IETF/ICANN Memorandum is in force, together with the Supplemental Agreement from January 1, 2014 (ICANN/ IETF Memorandum of Understanding Supplemental Agreement) ${ }^{4}$.

The peculiarity of the IETF/ICANN Memorandum is in its subjective components, as it is signed, from one side, by the ICANN Corporation - a legal entity of the state of California, USA, and from the other side by the IETF Group, which isn't a legal entity, and from the organizational and legal perspective is a part of the internal organizational system of the Internet Society (ISOC) - a legal entity of the Federal District of Columbia, USA, which was created in the organizational legal form of a non-profit corporation ${ }^{5}$.

The outstanding goal of the IETF/ICANN Memorandum is to define the technical works, which the IANA Administration is carrying out to fulfill the functions of the definition of the parameters of protocol used in the Internet standards of the IETF Group. According to the IETF/ICANN Memorandum, the ICANN Corporation, the IANA Administration, the ISOC Society and all the structural organizations working under the aegis of the ISOC Society participate in the implementation of the technical aspects of the IANA functions ${ }^{6}$. The IETF/ICANN Memorandum defines the

\footnotetext{
${ }^{4}$ URL: http://iaoc.ietf.org/documents/2014-ICANN-IETFMoU-Supplemental-Agreement-Executed.pdf

${ }^{5}$ See: D. C. Non-Profit Corporation Act. D. C. Columbia Corp. Code, Title 29. URL: http://www.venable.com/ files/Publication/1a3fb66e-357b-419e-829e-7ccb56e141d9/ Presentation/PublicationAttachment/23140013-bb80-473ba420-8aeeaf1f6170/combined_handouts_for_12-19_event.pdf (load date 09.08.2014).

${ }^{6}$ The organizations working under the aegis of the ISOC society are the IETF Group, the Internet Architecture Board (IAB), the Internet Engineering Steering Group (IESG), the Internet Research Task Force (IRTF), the Internet Research Steering Group (IRSG), the Request for Comments Editor (RFC).
} 
order of collaboration between the named organizations and structures while implementing the technical aspects of the IANA Administration work and in relation to the procedure of carrying out the functions of protocol parameter definition, used in the standards of the IETF Group, which reflect the existing mechanisms of the IANA functions implementation.

The legal contents of the IETF/ICANN Memorandum allows us to qualify it as a sub-contractor agreement, related to the ICANN/USA Government Contract. It should be noted that the IETF/ICANN Memorandum, as well as the ICANN/USA Government Contract, defines the "control" criterion while defining the circle of entities involved in the works by the IETF/ICANN Memorandum. The assignment of "the control" criterion in the ICANN/USA Government Contract is realized in the legal regulations of the IETF/ICANN Memorandum, and gets its practical application in the nature of parties of the organizations, enabling the technical implementation of the IANA functions, which are under the jurisdiction of the USA.

Undoubtedly, the implementation of the functions of the IANAis a key and critically important question in the trans-border governance of the technological infrastructure of the Internet, and the ICANN Corporation and its structural department, the IANA Administration, play a significant role in the institutional structure of the trans-border governance of the technological infrastructure of the Internet, as they are in fact the only contractors of the implementation of the IANA functions. The fact that the IANA functions are implemented in accordance with the ICANN/USA Government Contract testifies that the USA Government keeps control over the implementation of the functions of the IANA on a contractual, legal level. The working ICANN/USA Government Contract and the IETF/ICANN Memorandum related to it allow us to conclude that in the trans- border Internet governance the leading positions are taken by the legal institutions of the USA law, institutions working under the jurisdiction of the USA, or connected with the USA jurisdiction at a contractual legal level.

In fact, the quintessence of de lege lata (or "the law as it exists") of the current organizational and legal model of the trans-border governance of the technological infrastructure of the Internet is represented by the following, in some sense unique model: firstly, the implementation of the IANA functions, which is the key question in the trans-border governance of the technological infrastructure of the Internet; secondly, the centralized implementation of the IANA functions on a technological level by a "close circle" of institutions, either working under USA jurisdiction, or closely connected to the USA jurisdiction on the contractual legal level. Thirdly, the legal control of the USA Government assigned on the contractual legal level by the working ICANN/USA Government Contract and related to it by the IETF/ICANN Memorandum on the implementation of technical aspects of the IANA Administration the work of the IANA.

The above-stated remark makes it clear what the effect was that the NTIA announcement of March 14, 2014 about the USA Government plan had: to transfer the control of the implementation of the functions of the IANA to the Global Muti-stakeholder Community, and to change the governance of the technological infrastructure of the Internet radically ${ }^{1}$. The question of the need

\footnotetext{
${ }^{1}$ NTIA Announces Intention to Transfer Key Internet Domain Name Functions. - URL: http://www.ntia.doc.gov/pressrelease/2014/ntia-announces-intent-transition-key-internetdomain-name-functions; and also URL: press@ntia.doc.gov; URL: http://www.inta.org/INTABulletin/Pages/USDepartmentofCom merceAnnouncesIntenttoTransitionKeyInternetDomainNameF unctions.aspx. A number of experts say that the announcement of the USA Commerce Department wasn't a surprise for them. See for example: URL: https://www.afnic.fr/fr/ressources/blog/lelephant-iana-est-dans-la-salle-2.html; URL: http://www.afnic. fr/fr/ressources/blog/gouvernance-de-l-internet-au-travail.html); URL: http://arstechnica.com/tech-policy/2014/03/in-suddenannouncement-us-to-give-up-control-of-dns-root-zone/
} 
to change the current mechanism of the implementation of the functions of the IANA has been discussed more or less intensively for more than 15 years, both on national and international levels. However, many were taken aback by the NTIA announcement on March 14, 2014'. The brevity of the NTIA announcement of March 14, 2014 (a little bit over a page of text) does not remove the intrigue in both the document's appearance and the possible realization of its statements. If we analyze the contents of the NTIA Announcement sequentially from March 14, 2014, a few key moments should be singled out:

1. First of all, as a first step the NTIA Announcement of March 14, 2014 contains the address from the NTIA Administration to the ICANN Corporation to organize the discussion with all the stakeholders interested in the change of the current governance mechanism, coordination of the DNS system and the role of the USA Commerce Department's NTIA Administration. According to the NTIA Announcement of March 14, 2014, it is the ICANN Corporation that controls all the discussion and proposition development process with the participation of the stakeholders, and it should also develop the respective propositions. The principal factor is that the propositions developed by stakeholders should be approved and accepted by the USA Government.

In fact, the USA Government has chosen the safest algorithm of collaboration with the stakeholders - via the ICANN Corporation,

\footnotetext{
${ }^{1}$ The heated discussion of this matter can be seen in the article titles and comments "September 30, 2015 - Day of Internet liberation from the USA Government","Withdrawal of troops from IANA", "The USA Government opens a new page in Internet governance". - URL: http://thegovlab. org/the-govlab-scan-special-issue-reactions-to-the-ntiaannouncement-on-globalizing-iana-functions/; URL: http:// www.ripe.net/internet-coordination/internet-governance/ internet-technical-community/iana/iana-oversight-transition; URL: http://arstechnica.com/tech-policy/2014/03/in-suddenannouncement-us-to-give-up-control-of-dns-root-zone/
}

the legal institution of the USA law, connected to the USA Government by contractual legal relations. With such an algorithm, the expanding of the subjective and functional competence of the ICANN Corporation is quite predictable, and it is hard to evaluate such a development of actions unambiguously. Firstly, at the moment the USA Government's desire to "lock" the conversation on the ICANN Corporation, giving it the functions of control over the "transition period" execution is perceived quite neutrally by stakeholders, including international organizations and the governments of other countries. Even if the competence of the ICANN Corporation will be changed, the process of the internationalization of the domain zone, the new TLG, the mechanisms of the international control over the ICANN Corporation activities etc. - will be developing in one way or another. The Internet community would hardly downgrade the work of an organization not accountable to anyone, while the accountability of the ICANN Corporation to the Internet community is being preserved by the Affirmation of Commitments by the United States Department of Commerce and the Internet Corporation for Assigned and Numbers ${ }^{2}$, which is infinite. Secondly, it is the action of the above-mentioned agreement and its infinite quality that also preserves the "infinite quality" of the relations of the ICANN Corporation with the USA Government from one side, and the accountability of the ICANN Corporation to the Internet community from the other side. Thirdly, the position of the ICANN Corporation trying to preserve the status quo of the existing architecture of the governance of the technological infrastructure of the Internet, and not supporting the creation of any other organization, is quite logical. Creating any new organization will potentially involve a "conflict of interests

\footnotetext{
${ }^{2}$ URL: http://www.icann.org/ru/news/announcements/ announcement-30sep09-ru.htm (load date 09.08.2014).
} 
and "a conflict of competences", especially because the work of any organization should reflect the "multistakeholder" approach ${ }^{1}$.

2. The NTIA Announce from March 14, 2014 pays attention to the fact that the current mechanism of the implementation of the functions of the IANAwas built historically and the contractual legal basis for it is the ICANN/USA Government ${ }^{2}$ Contract, time of which comes to an end on September 30, 2015. However, the "termination process" of the contractual legal relations by this contract is not "irreversible". In the contractual legal context the USA Government reserves the possibility to control the implementation of the functions of the IANAeven after September 30,2015, because, as noted earlier, the ICANN/USA Government Contract includes the seven year prolongation option.

3. It is of substantial importance that the USA Government is not considering the possibility of discussing any propositions from the stakeholders concerning the creation of a new control mechanism for the implementation of the functions of the IANA, if the control functions are passed to a group of countries or an international intergovernmental organization. With the absence of real mechanisms to "force" the USA Government to "give up control over the implementation of the functions of the IANA, the "non-approval" of the propositions developed by stakeholders or the withdrawal of the USA Government in any new mechanism will inevitably impact on the legitimacy of the whole transition pro-

\footnotetext{
${ }^{1}$ On March 14, 2014 the ICANN Corporation published a document called ICANN Public Consultation Processes, which describes temporary timetable of the meetings to discuss the mechanisms and the order of transition of IANA functions implementation to the stakeholder community, which provides for seven stages of the meetings within the ICANN sessions (ICANN sessions 49-53) - URL: https://www.icann.org/en/ system/files/files/functions-transfer-process-14mar14-en.pdf

${ }^{2}$ See also Cooperative Agreement to Perform Related Root Zone Management Functions between ICANN Corporation and VeriSign Inc.
}

cess of the implementation of the functions of the IANA to the global multistakeholders' community.

The USA Government is not changing its position concerning the fact that no government in the world, as well as no international intergovernmental organization, would be able to not only provide the implementation of the functions of the IANA, but also to make this process legitimate. Such a position has some legal grounds, which are referred to in the NTIA Announcement from March 14, 2014. The question is that the legal acts currently in force were separately passed by both chambers of the USA Congress. Specifically the two legal acts were: The Concurrent Resolution of the USA Senate No.50 from December 5, 2012 ${ }^{3}$ and of the House of Representatives of the USA No.127 from September 10, 2012 — “The Concurrent Resolution, Expressing the Sense of Congress Regarding Actions to Preserve and Advance the Multistakeholder Governance Model Under Which the Internet has Thrived".

The inevitable consequences of the current situation is that the "control" of the transition period is done by the ICANN Corporation, and till the ICANN finishes this process, the role of the USA Government will remain unchanged till September 30, 2015, and possibly in the future, if the proposed mechanism, developed by the stakeholders, is not accepted and approved by the USA Government.

4. The NTIA Announcement from March 14, 2014 provides that during the development of propositions, which the ICANN Corporation should present to the USA Government, the stakeholders should take into account the following four key principles:

\footnotetext{
${ }^{3}$ URL: http://democrats.energycommerce.house.gov/sites/ default/files/documents/Congressional-Record-S-ConRes-50-2012-12-5.pdf

${ }^{4}$ URL:https://www.govtrack.us/congress/bills/112/hconres127/text
} 
- the support and strengthening of the multistakeholder model for Internet governance;

- the maintaining of the security, stability and resiliency of the DNS system on the Internet;

- the serving of the needs and expectations of global consumers and partners of the IANA services; and,

- the maintenance of the openness of the Internet.

5. The NTIA Announcement of March 14, 2014 clearly defines the subjective components of the institutions, in cooperation with which the ICANN Corporation should hold the transition procedures, related to the transition of the control over the implementation of the IANA functions: the IETF Group, the Internet Architecture Board (IAB), the Internet Society (ISOC), the Regional Internet Registries (RIRs), the Top Level Domain Name Operators ${ }^{1}$, and VeriSign Inc. Corporation.

The NTIA Announcement of March 14,2014 clearly highlights the precedent connected with the creation of the ICANN Corporation in 1998, when the USA Government changed its administrative role in the DNS system and passed these functions to the private sector representing the corporation. More than that, in the NTIA Announcement on March 14, 2014 it is clearly noted that the whole creation of the ICANN Corporation reflected the position of the USA Government about the temporary nature of its control role over the implementation of the functions of the IANA. From the idea of the NTIA Announcement of March 14, 2014, the upcoming radical change in the governance of the technological infrastructure of the Internet due to the USA Government's intentions to transfer

${ }^{1}$ URL:http://dir.yahoo.com/computers_and_internet/internet/ domain_name_registration/top_level_domains_ttds_/ registry_operators/international_country_codes/ the control of the implementation of the functions of the IANA to the global multistakeholder community, will conclude the process started by the USA Government in 1998.

We should pay attention to the fact that such a precedent is acceptable and possible. However, during the last 16 years the role of governments and international intergovernmental organizations the UN, the International Telecommunication Union etc.) has changed significantly, and it can be defining to the format of current processes and to the decision-making of other stakeholders, which represent the private sector, the business community, civil society, and the technical and academic community.

Concluding the above-mentioned, and leaving aside the perspective of the search for de lege ferenda (ie with a view to the future law) format of trans-border Internet governance, we should note that the realization of the NTIA Announcement on March 14, 2014 is objectively related to the need to answer the questions about how the legitimacy of the transition process of the implementation of the functions of the IANAto the "global multi-stakeholders community" will be attained; who will control the technical aspects of the implementation of the functions of the IANA; which mechanisms will be realized to provide the control of the adequacy of the implementation of the functions of the IANA, etc. The solution to these questions is in one way or another the essence of trans-border Internet governance.

\section{II.}

The NTIA Announcement of March 14, 2014 was definitive for the Global Multistakeholder Meeting on the Future of Internet Governance, see - "NET-Mundial 2014". The outcome document of the NETMundial 2014 is the NET-Mundial Executive 
Stakeholder Committee (EMC) Outcome Document of April 3, 2014․

Two questions were included in the agenda of the NET-Mundial 2014, which are connected to the future of trans-border Internet governance: the first question is the future of Internet governance; the second is the Roadmap for the Future Evolution of the Internet Governance Ecosystem.

The key principles of Internet governance, which form the basis of the global, multistakeholder, effective, legitimate and developing model of Internet governance, are distributed among five key blocks in the the document which was produced by the NET-Mundial 2014: human rights; Internet infrastructure; access to the Internet; Internet governance; Internet standards.

1). The principles connected to human rights are the key value, which should be the basis for all aspects of Internet governance. The rights that humans have offline" should be protected "online" as well, according to the key human rights conventions, such as the Universal Declaration of Human Rights and the International Covenant on Economic, Social and Cultural Rights.

The human rights include the following, non-comprehensive, list of rights: the right to access information, and exchange it freely; the right of reunions; the right to express one's opinion in the Internet, and the right to send and receive information via the Internet with no third parties involved; the right to confidentiality, meaning confidentiality in both online and offline, including the ban on the illegal distribution of personal data, and "shadowing"; Internet accessibility to physically challenged persons, and their access to all online resources; cultural

\footnotetext{
${ }^{1}$ URL: https://wikileaks.org/netmundial-outcome/http:// content.netmundial.br/contribution/panel-on-global-internetcooperation-and-governance-mechanisms-contribution-tonetmundial/204 (load date 09.08.2014); and also -URL: http:// netmundial.br/wp-content/uploads/2014/04/NETmundialMuti-stakeholder-Document.pdf (load date 09.08.2014).
}

and language diversity and non-discrimination; Internet evolution for the achievement of global evolution goals, agreed upon at an international level.

2 ). The Internet infrastructure should remain stable, safe, flexible and reliable. The effectiveness of Internet governance is defined by the constant cooperation of different stakeholders. The principles of stability, resiliency and flexibility of the Internet should become key for all Internet governance stakeholders.

$3)$. Access to the Internet is ensured by Internet integrity and its non-fragmentation. The Internet should remain an open and accessible space, including the body of unique identifiers, and with the ability to perform operations on a global scale. The open and distributed architecture of the Internet supports access to any information, software and services at the user's choice. The access rules for the Internet should be technologically neutral, have the ability to implement new technologies and various types of Internet usage.

4). Internet governance should be open: it should be carried out with the participation of all stakeholders; it should be neutral, taking into account human rights, and based on the principle of accountability to the Internet community. Stakeholders' participation in the Internet governance should reflect not only the interests and approaches of the stakeholders, but also their participation in all processes, related to Internet governance.

5). The Internet standards are the basis of Internet functioning, and they should maintain the Internet's uniqueness so that it remains accessible, flexible, stable, decentralized, safe and open.

The Roadmap for the Future Evolution of the Internet Governance Ecosystem formulates the key goal: the enhancement of the structure of existing Internet governance, enabling the participation of all 
stakeholders. The Roadmap defines that the structure of Internet governance is a controlled ecosystem, which includes various organizations. The enhancement of the existing structure of Internet governance should be open, accessible, and the process itself should be accountable to the Internet community. We should officially acknowledge the values of the Internet governance model, which involves all stakeholders, and the model of "all stakeholders' participation" should be reinforced and enhanced. The participation of stakeholders in Internet governance should reflect geographical balance, and include representatives of developing countries and communities.

The Roadmap formulates questions, which need to be resolved by the Internet community in the process of the enhancement of the multistakeholder governance model. Among others, they include the questions related to the institutional enhancement of Internet governance. In particular, it is suggested to reinforce the Internet Governance Forum (IGF); to extend the time of its functioning, and in future to make it a permanently working organ; to ensure guaranteed and stable financing of the Internet Governance Forum. On the institutional level, it is planned to preserve the format of the NET-Mundial 2014, and to see it as a "platform for the extended dialogue between stakeholders, outside the boundaries of Internet Governance Forum".

The Roadmap expresses support of the NTIA Announcement of March 14, 2014 in regard to the changes of the USA Government administrative role in Internet governance, and it points out that any newly-created mechanism should on the one hand defend the open and accessible basis of Internet governance decision- making, ensuring the stability and universality of the Internet, and on the other hand should create a sensible distribution between the processes of governance decision-making and the tech- nological aspects of Internet governance. The Roadmap reflects questions related to particular aspects of Internet governance, and also formulates the problems set that need to be discussed outside the boundaries of the NET-Mundial 2014, namely: the role and responsibilities of stakeholders within the ecosystem of Internet governance, including the formation of a legal base; questions for jurisdiction and their impact on Internet governance; the creation of a Code of Conduct based on principles defined in the Roadmap, with the aim of ensuring proper Internet governance.

\section{III.}

In May 2014, the Panel on Global Internet Cooperation and Governance Mechanisms ${ }^{1}$ finished its work, see - "The Panel on Global Internet Cooperation". The Panel on Global Internet Cooperation was acting as an organizational structure, including experts, various stakeholder groups, representing the governments, civil society, the private sector, technical society and international organizations. The subject domain of the Panel on Global Internet Cooperation work was related to the analysis of the evolution and the extension of the boundaries of Internet governance ${ }^{2}$.

In May 2014, the Panel on Global Internet Cooperation presented its summary document - the Roadmap of the new approach to the future of Internet governance in the form of report Towards a Collaborative, Decentralized Internet Governance Ecosystem ${ }^{3}$, see the Report of

\footnotetext{
${ }^{1}$ URL: http://internetgovernancepanel.org (load date 09.08.2014).

2 Panel on Global Internet Cooperation and Governance Mechanisms was organized by ICANN Corporation and the World Economic Forum with the support of Annenberg Foundation-URL: http://internetgovernancepanel.org/history (load date 09.08.2014).

${ }^{3}$ URL: http://internetgovernancepanel.org/sites/default/files/ ipdf/XPL_ICAN1403_Internet\%20Governance\%20iPDF_06. pdf (load date 09.08.2014).
} 
the Panel on Global Internet Cooperation. In the Report of the Panel on Global Internet Cooperation two key factors were defined as starting points, namely: The NTIA Announcement of March 14, 2014 and the summary document of the NET-Mundial 2014, approved on April 3, 2014

The Report of the Panel on Global Internet Cooperation is quite a big document, which reflects the key principles and evolution parameters of the global Internet governance ecosystem, and also gives a plan and timetable for Internet cooperation between all stakeholders. The important point is that the Report of the Panel on Global Internet Cooperation totally supports and accepts the principles and evolution parameters of the global Internet governance ecosystem, which were defined in summary documents of the NET-Mundial 2014, as the basis of Internet governance, and the summary documents of the NETMundial 2014 make up an integral part of the Report of the Panel on Global Internet Cooperation

The provisions of the Report of the Panel on Global Internet Cooperation, related to the institutionalization process of the Internet governance ecosystem are substantial. Firstly, the critical provision is that the ecosystem of Internet governance should remain decentralized. Secondly, the Report of the Panel on Global Internet Cooperation provides the exact measures needed for the evolution of the global Internet governance ecosystem till 2017, including: the broad participation and cooperation of all stakeholders; the development of new, and the reinforcement of existing mechanisms of Internet governance; cooperation in decision-making, related to Internet governance; the resolution of the question of the financing of the resource base for Internet governance ecosystem; the support of accountability by the ICANN Corporation to the Internet community, and the interna- tionalization of the implementation of the functions of the IANA, etc.

Thirdly, the Report of the Panel on Global Internet Cooperation defined that the key institutionalization component of the decentralized Internet governance ecosystem is the Distributed Governance Groups ${ }^{1}$. It is also noted that the work of governance groups should be based on the principles of Internet governance set in the outcome documents of the NET-Mundial 2014.

The Report of the Panel on Global Internet Cooperation defined that the institutional structure of Internet governance is formed in a specific field of governance, and includes specific governance groups. For example, it named the governance groups, which make up the institutional structure of Internet governance in the field of IPaddresses, including the Regional Internet Registries (RIR); the ICANN Corporation and its structural parts, such as the Address Supporting Organizations (ASO) and the Internet Assigned Numbers Authority (IANA); the Number Resource Organization (NRO); and Internet Service Providers (ISPs).

Fourthly, the Report of the Panel on Global Internet Cooperation determines that the fundamental parameters of Internet governance ecosystem should correlate with the technological architecture of the Internet. In particular, it outlines three components: the Distributed component. Internet technology based on a distributed system, allows many entities to create and use the variety of structures and governance systems. Further, the Participatory component, allows the participation of all the stakeholders in the formation of standards and policies; the Layered component - layered nature of the technological infrastructure of Internet, related to the fact that the

\footnotetext{
${ }^{1}$ URL: http://internetgovernancepanel.org (load date 09.08.2014).
} 
Internet governance ecosystem covers lo$\mathrm{cal} /$ national, regional and global governance levels, allowing for optimal decision-making at each governance level.

In such a way, the Report of the Panel on Global Internet Cooperation clearly brings in a conceptual clarity concerning the need for keeping and developing the historicallybuilt Multistakeholder model of Internet governance. More than that, the Report of the Panel on Global Internet Cooperation evaluated this model as the basis for forming the Internet governance ecosystem and the institutionalization of its trans-border governance.

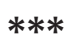

It is quite difficult to evaluate the perspectives of Internet evolution unambiguously, as well as its trans-border governance. At the same time, the trans-border Internet governance and the process of its institu- tionalization will undoubtedly be defined by the above-noted events, and by the realization of measures, defined in corresponding documents. And even if the status quo of the existing architecture of technological infrastructure governance of Internet remains and the implementation of the IANA functions is formally preserved by the ICANN Corporation, the internationalization of domain zone, the new TLG, the international governance mechanisms over the work of the ICANN corporation etc. - will be developing in one way or another. Besides, it is of major importance now to keep the dialogue about the Internet governance ecosystem, which is outlined on high-level by the summary documents of the NETMundial 2014, formulating the principles of Internet governance and the Roadmap of the future evolution of the Internet governance ecosystem.

\section{References (transliterated)}

1. D. C. Non-Profit Corporation Act. D. C. Columbia Corp. Code, Title 29. URL: http:// www.venable.com/files/Publication/1a3fb66e-357b-419e-829e-7ccb56e141d9/ Presentation/PublicationAttachment/23140013-bb80-473b-a420-8aeeaf1f6170/ combined_handouts_for_12-19_event.pdf (load date 09.08.2014) 